\title{
Developing a tool for the validation of quantitative DCE-MRI
}

\author{
Karin Bol ${ }^{\mathrm{abc}}$, Joost C. Haeck ${ }^{\mathrm{abc}}$, Lejla Alic ${ }^{\mathrm{ab}}$, , Monique Bernsen ${ }^{\mathrm{b}}$, Marion de Jong, \\ Wiro J. Niessen ${ }^{\text {abd }}$, Jifke F. Veenland ${ }^{\text {ab }}$ \\ ${ }^{\mathrm{a} B I G R}$ - Biomedical Imaging Group Rotterdam, Departments of ${ }^{\mathrm{b}}$ Radiology and ${ }^{\mathrm{c}}$ Nuclear Medicine, \\ Erasmus MC, Rotterdam, P.O. Box 2040, 3000 CA Rotterdam, The Netherlands; \\ ${ }^{\mathrm{d}}$ Faculty of Applied Sciences, Delft University of Technology, Delft, The Netherlands
}

\begin{abstract}
Dynamic Contrast-Enhanced Magnetic Resonance Imaging (DCE-MRI) is becoming an indispensable tool to noninvasively study tumor characteristics. However, many different DCE-analysis methods are currently being used. To compare and validate different methods, histology is the gold standard. For this purpose, exact co-localization between histology and MRI images is a prerequisite. In this study a methodology is developed to validate DCE-data with histology with an emphasis on correct registration of DCE-MRI and histological data. A pancreatic tumor was grown in a rat model. The tumor was dissected after MR imaging, embedded in paraffin, and cut into thin slices. These slices were stained with haematoxylin and eosin, digitized and stacked in a 3D volume. Next, the 3D histology was registered to exvivo SWI-weighted MR images, which in turn were registered to in-vivo SWI and DCE images to achieve correct colocalization. Semi-quantitative and quantitative parameters were calculated. Preliminary results suggest that both pharmacokinetic and heuristic DCE-parameters can discriminate between vital and non-vital tumor regions. The developed method offers the basis for an accurate spatial correlation between DCE-MRI derived parametric maps and histology, and facilitates the evaluation of different DCE-MRI analysis methods.
\end{abstract}

Keywords: DCE-MRI, histology, registration, DCE-analysis, quantitative, semi-quantitative, pharmacokinetic modeling, two-compartment model

\section{INTRODUCTION}

In oncology, Dynamic Contrast-Enhanced Magnetic Resonance Imaging (DCE-MRI) ), in which the signal intensity over time is measured following contrast injection, is frequently used as a tool to detect tumors or metastases, to monitor tumor growth and to assess the response to anti-tumor treatment. Although many different analysis methods have been used to quantify tumor characteristics from DCE-MRI data, a standard analysis method has not yet been adopted.

Both semi-quantitative and quantitative analysis methods can be employed to analyze the signal intensity time curves (SI-curves) in DCE-MRI ${ }^{1}$. A semi-quantitative model derives parameters from the SI-curves such as the area under the curve (AUC), slope of wash-in and wash-out of the contrast agent and maximal enhancement. The semi-quantitative model is straightforward and easy to implement. However, derived parameters do not necessarily have a physical correlation with the underlying tumor characteristics and often are a mixture of several quantitative parameters $/$ measures. Furthermore, it is difficult to compare measurements from scans acquired at different times or from different scanners, owing to the lack of an absolute intensity scale. Quantitative analysis methods are based on pharmacokinetic modeling, assuming that the contrast is divided into several compartments. Analysis using quantitative models is less straightforward as additional information is required, such as a T1-map and knowledge of the Arterial Input Function (AIF).

For the validation of DCE-MRI derived parameters, histology is still the gold-standard. The haematoxylin and eosin stain $(\mathrm{H} \& \mathrm{E})$ is commonly used to discriminate viable from necrotic tumor tissue. However, not all studies show a correlation between DCE-parameters and histology ${ }^{2-6}$. One of the factors influencing this correlation could be the poor colocalization of the histology with the MRI slices, which has not received much attention ${ }^{1}$. Also changes in morphology (shrinkage) and orientation once specimens are dissected and placed in formalin fixative may play a role ${ }^{7,8}$. In view of this, the goal of this study is to develop a novel methodology for validating DCE-MRI derived parameters, by achieving a more accurate co-localization between histology and DCE-MRI images. Hereto, a multi-step registration process involving an intermediate ex-vivo MRI scan will be used ${ }^{9}$.

Medical Imaging 2011: Biomedical Applications in Molecular, Structural, and Functional Imaging, edited by John B. Weaver, Robert C. Molthen, Proc. of SPIE Vol. 7965, 79651 · ( ) 2011 SPIE · CCC code: $0277-786$ X/11/\$18 - doi: 10.1117/12.877834 


\section{METHODS}

\subsection{Animal and tumor model}

For this study a male Lewis rat (Harlan_CPB, Austerlitz, The Netherlands), weighing approximately 300 grams, was used with approval of the Committee for Animal Research of the Erasmus MC, Rotterdam, The Netherlands. Pancreatic tumor cells (CA20948 tumor) were inoculated subcutaneously in the right hind limb. The tumor was imaged when it reached a diameter of $1.5-2 \mathrm{~cm}$. The animal was anesthetised before imaging using Isoflurane (Nicholas Pyramal Limited, London, UK). After completing the examinations the animal was sacrificed.

\subsection{DCE-MRI Imaging protocol}

A clinical 3 Tesla MRI scanner (Signa Excite, GE Healthcare, USA) was used for imaging. All images were acquired using the body coil. DCE images were obtained using TRICKS (Time Resolved Imaging of Contrast Kinetics), for achieving high temporal resolution. T1-weighted MR images were used for calibration of the DCE data and SWIweighted MR image were used for registration purposes (see also figure 1 in section 2.4.1). In-vivo MRI settings: T1weighted MRI: $\mathrm{Tr} / \mathrm{Te}=20 / 4 \mathrm{~ms}$, flip angels of $3,5,10,15,20,35$ and $50^{\circ}$ and a XYZ resolution of $0.2 \times 0.2 \times 0.7$; SWIweighted MRI: $\mathrm{Tr} / \mathrm{Te}=24.4 / 12.0 \mathrm{~ms}$, flip angel of $10^{\circ}$ and a $\mathrm{XYZ}$ resolution of $0.098 \times 0.098 \times 0.20$; TRICKS DCE-MRI settings: $\mathrm{Tr} / \mathrm{Te}=5.44 / 1.46 \mathrm{~ms}$, flip angle of $10^{\circ}, \mathrm{XYZ}$ resolution of $0.2 \times 0.2 \times 0.7$ and a temporal resolution of $4.13 \mathrm{~ms}$. Exvivo MRI settings: SWI-weighted MRI: $\mathrm{Tr} / \mathrm{Te}=23.5 / 8.9 \mathrm{~ms}$, flip angel of $10^{\circ}$ and a XYZ resolution of $0.098 \times 0.098 \times 0.40$.

\subsection{Histology}

After in-vivo MR imaging the tumor was dissected and dyed in order to track its orientation with respect to the in-vivo situation. After dissection the tumor was placed in $200 \mathrm{cc} 10 \%$ buffered formalin (Boom, The Netherlands) for fixation purposes. When the fixation process was completed, a thin section of the tumor was sliced off along the longest tumor axis in order to create a reference plane for ex-vivo MRI, thereby defining the plane for histological sectioning. The tumor was washed and embedded into agar to prevent tumor movement and to provide a water rich environment for exvivo MRI. After the ex-vivo MR imaging, the tumor was embedded in paraffin. Sections of $4 \mu$ m at approximately 80 $\mu \mathrm{m}$ intervals were cut using a microtome. These sections were fixed on a glass slide and stained with haematoxylin and eosin (H\&E). The slides were digitized using the NanoZoomer (C9600. Hamamatsu, Japan) with a magnification of 20 and a resolution of $3.64 \mu \mathrm{m}$.

\subsection{Data analysis}

\subsubsection{Registration DCE-MRI with histology}

In order to match histology to DCE-MRI, the registration scheme of Alic et al. ${ }^{9}$ was used. In short this method consists of two steps: A) registration of 3D stacked histology to 3D ex-vivo SWI-weighted MRI, and B) registration of 3D exvivo SWI-weighted MRI to 3D in-vivo MRI. (figure 1). The 3D histology volume was created by stacking the digitized histology slices using a rigid registration scheme. Then the registration of histology to ex-vivo and ex-vivo to in-vivo was achieved using third order non-rigid B-splines with mutual information as similarity measure to be optimized. The separate transformations were subsequently concatenated in order to accomplish co-registration of 3D histology to invivo MRI. All registrations were performed using Elastix ${ }^{10}$ (http://elastix.isi.uu.nl/). 

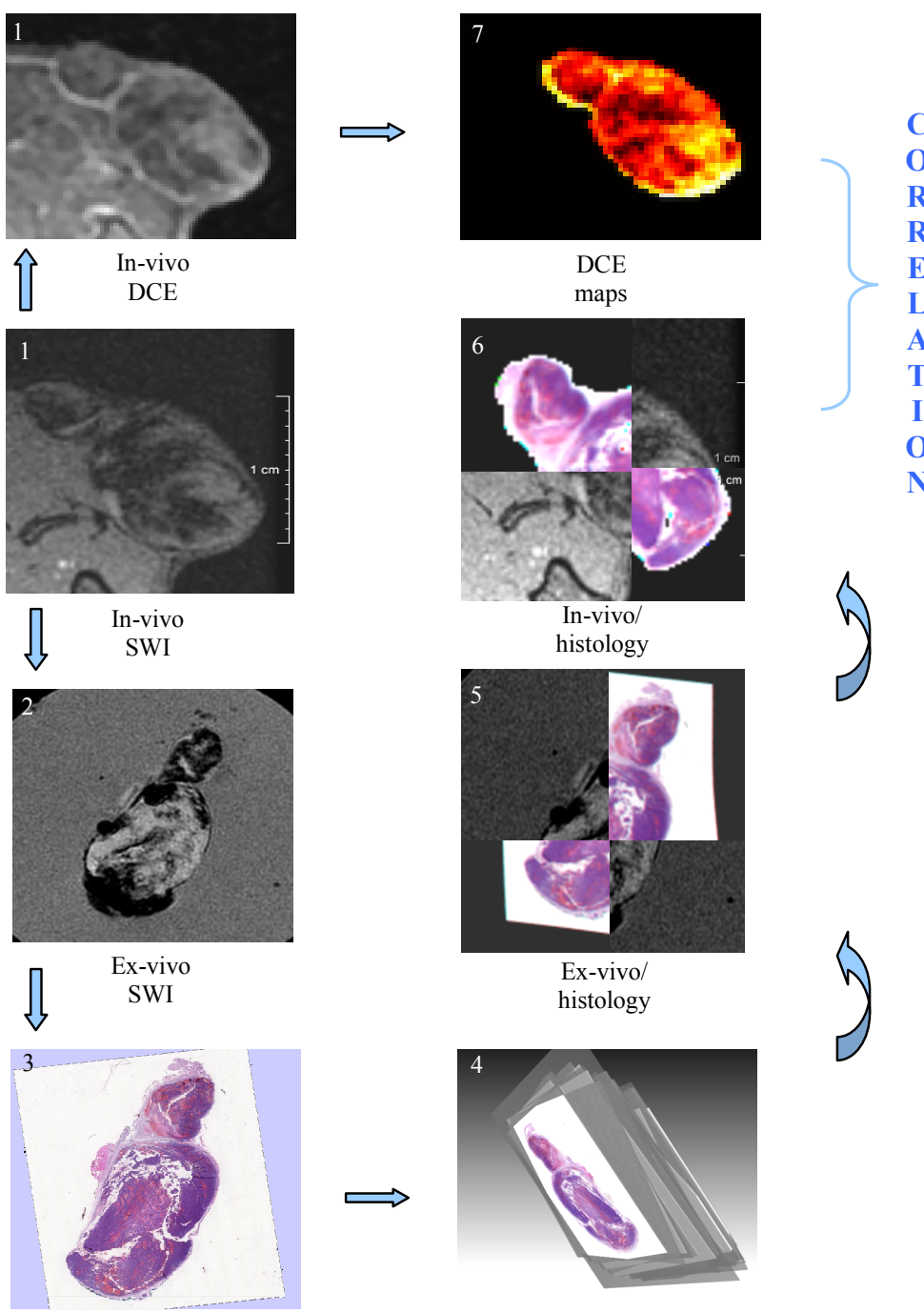

Ex-vivo/ histology

Histology

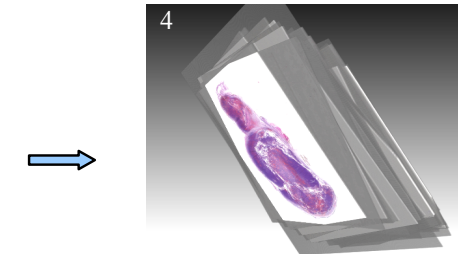

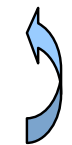

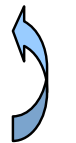

3D stack

Figure 1; Different steps to achieve registration between 3D histology and DCE-MR images: 1) in-vivo SWI to DCE-MRI registration, 2) ex-vivo SWI, 3) histology data, 4) stacking histology slices into a 3D volume, 5) registering histology to ex-vivo SWI, 6) registering ex-vivo SWI/histology to in-vivo SWI/DCE, 7) calculating DCE-parameter maps.

\subsubsection{DCE-MRI quantification}

The semi-quantitative parameters (i) maximum enhancement (Smax), (ii) area under the curve (AUC), (iii) wash-in and (iv) wash-out were calculated from the SI-curve. Next this curve was converted into a contrast-enhancement curve using equation (1):

$$
C(t)=\frac{1}{\alpha}\left(\frac{1}{T_{1}(t)}-\frac{1}{T_{1}(0)}\right)
$$

with $\mathrm{C}(\mathrm{t})(\mathrm{mM})$ representing the concentration of the contrast agent at time $\mathrm{t}, \alpha$ the relaxivity (mM/s) of the contrast agent (Gadovist; $\alpha=3.6$ ), $\mathrm{T}_{1}(\mathrm{t})$ the $\mathrm{T} 1$-value of the tissue at time $\mathrm{t}$ after injection of the contrast agent and $\mathrm{T}_{1}(0)$ the 
T1-value of the tissue before contrast injection. The latter was calculated using a linearized T1-mapping method ${ }^{11}$. Quantitative parameters were calculated by fitting a two-compartment model (originally proposed by Brix et.al ${ }^{12}$, figure 2 ) to the contrast-enhancement curve.

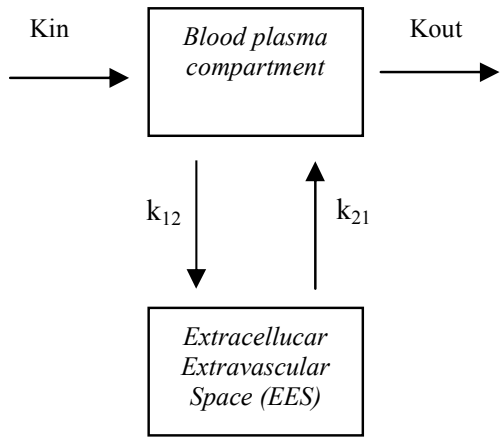

Figure 2; Two-compartment model used to calculate quantitative DCE-MRI parameters

This two-compartment model was solved by using a Laplace transform ${ }^{13}$, where the bolus of the arterial input function (AIF) is assumed to be an instantaneous injection of contrast agent and thus modeled as a delta function. The derived equation becomes:

$$
\begin{gathered}
C(t)=\frac{A}{a+b}\left(\exp ^{-b t}-\exp ^{-a t}\right) \\
a=\frac{k_{1}+\sqrt{k_{1}^{2}-4 k_{2}}}{2} \quad b=\frac{k_{1}-\sqrt{k_{1}^{2}-4 k_{2}}}{2} \\
k_{1}=k_{\text {out }}+k_{12}+k_{21} \quad k_{2}=k_{21} * k_{\text {out }} \quad A=\frac{V_{c} k_{12}}{V_{p}}
\end{gathered}
$$

with $\mathrm{k}_{12}$ a measure of influx of contrast agent into the tumor (also known as $\mathrm{k}_{\text {trans }}$ ) and $\mathrm{k}_{21}$ the out flux of contrast agent $\left(\mathrm{k}_{\mathrm{ep}}\right)$. Parameters $\mathrm{a}$ and $\mathrm{b}$ represent a combination of $\mathrm{k}_{12}, \mathrm{k}_{21}$ and $\mathrm{K}_{\text {out }}$. Vc and $\mathrm{Vp}$ are the volumes of the central-blood plasma compartment and peripheral EES respectively, and $\mathrm{t}$ is the time passed since injection.

\subsubsection{Validating DCE-MRI with histology}

In order to validate the different DCE parameters, vital and non-vital regions were selected by drawing regions of interest (ROI's) on histology slices in the 3D volume. These regions were then transformed to the parametric maps, using the transformation parameters resulting from the registrations.

\section{RESULTS}

An example of derived DCE-MRI parametric maps is shown in figure 3. As can be seen from the r-square values in the figure (upper right), the two-compartment model used here does not always results in a good fit. Vital (black) and nonvital (gray) regions of interest were drawn in the registered histology slice. For these regions mean and standard deviation were calculated in the subsequent DCE parameter maps (table 1). Some of the standard deviations are considerable, however, a two-tailed t-test performed between the vital and non-vital regions per parameter showed that all difference were significant ( $p$-value $<<0.05$ ). Scatter plots were made to investigate whether the DCE parameters allow for segmentation between vital and non-vital regions (figure 4). It seems that segmentation is possible using a combination of two parameters. 


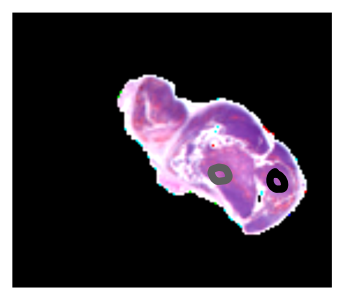

Histology

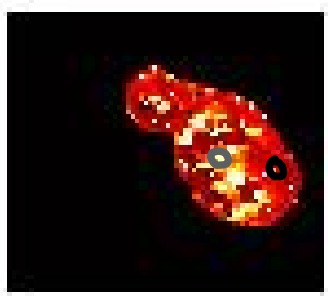

Parameter a

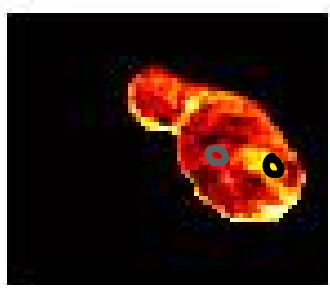

Smax

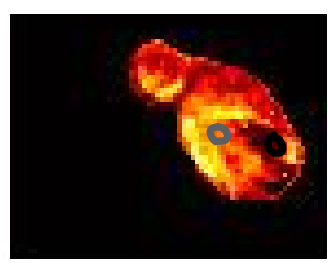

Wash-out

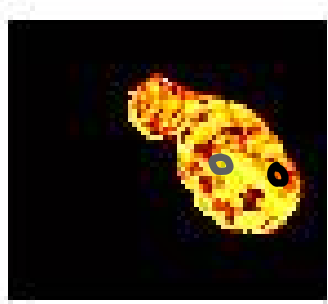

r-square

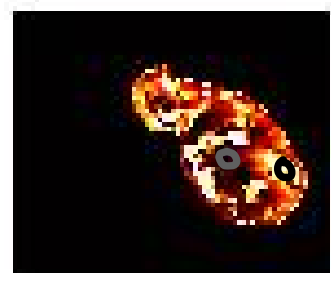

Parameter b
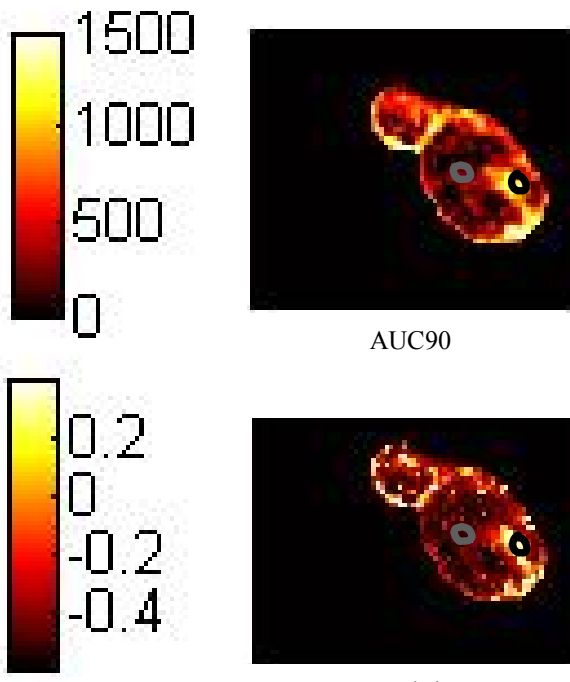

AUC90

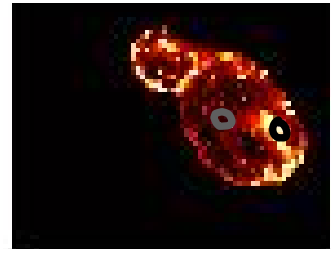

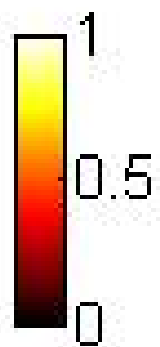
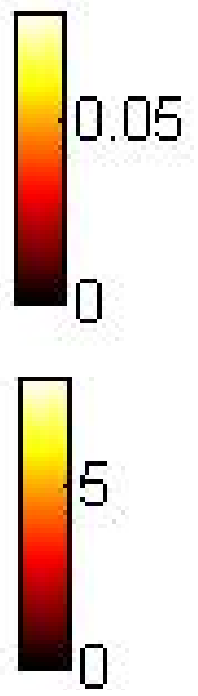

Wash-in

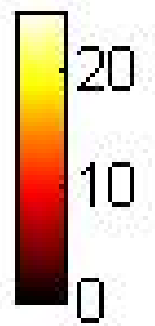

Figure 3: An example of DCE-MRI parametric maps, with at the top left the registered histology slice. The black circle denotes a vital region of interest and the gray circle denotes a non-vital region. 

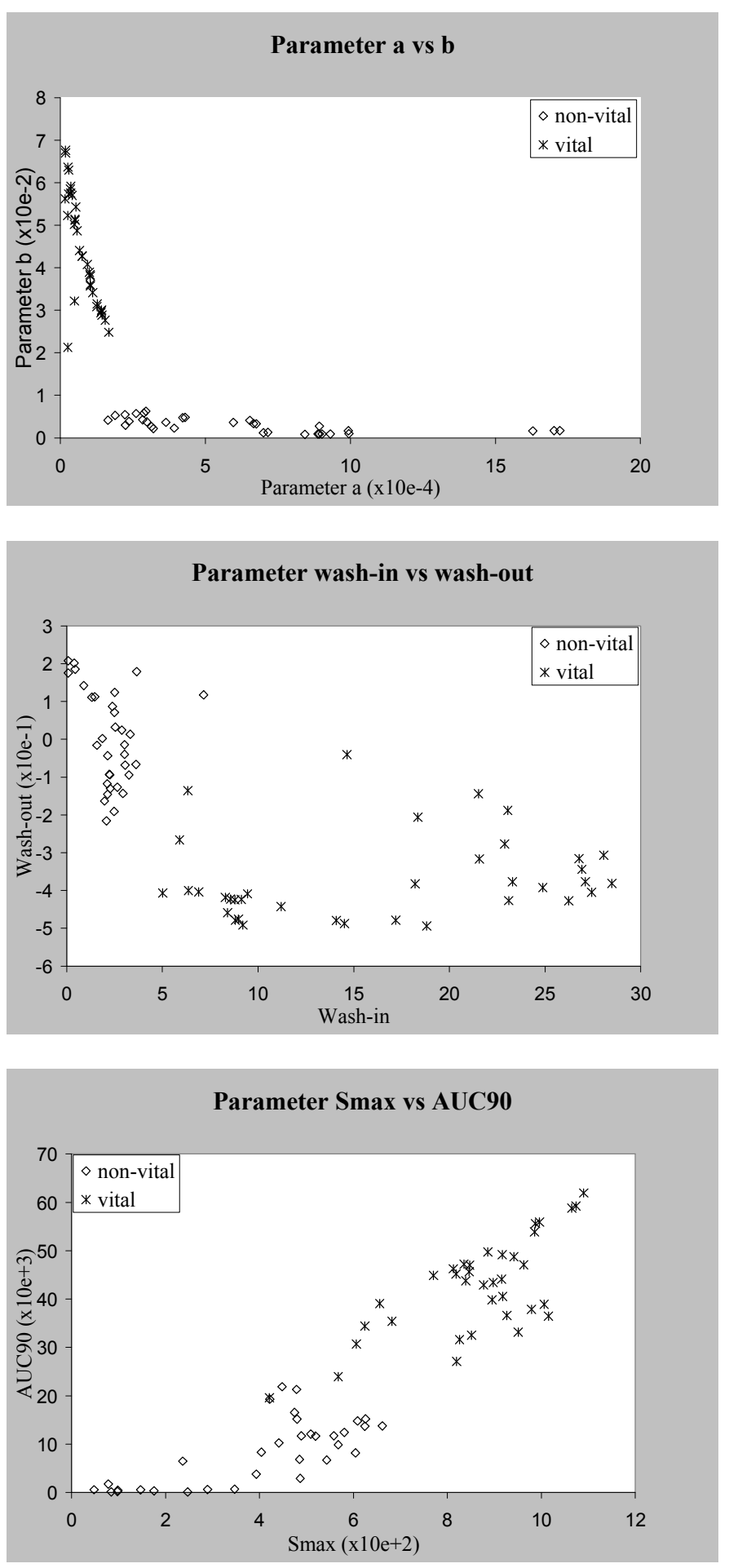

Figure 4; Scatter plots for the different DCE parameters of pixel-values in the vital and non-vital regions of interest 
Table 1; Mean and standard deviation (sd) of vital and non-vital regions of interest for different DCE parameters

\begin{tabular}{||l|r|r|r|r|r||}
\hline \hline \multirow{2}{*}{ PARAMETER } & \multicolumn{2}{|l|}{ NON-VITAL ROI } & \multicolumn{2}{l|}{ VITAL ROI } & \multicolumn{1}{l||}{ T-TEST } \\
\cline { 2 - 6 } & Mean & sd & Mean & p-value \\
\hline a (x10E-4) & 6.4 & 4.31 & 0.75 & 0.46 & $<0.05$ \\
\hline b (x10E-2) & 0.31 & 0.17 & 4.42 & 1.32 & $<0.05$ \\
\hline r-square & 0.81 & 0.05 & 0.71 & 0.09 & $<0.05$ \\
\hline Smax (x10E+2) & 4.02 & 1.87 & 8.64 & 1.49 & $<0.05$ \\
\hline AUC90(x10E+3) & 8.49 & 6.78 & 42.46 & 9.91 & $<0.05$ \\
\hline wash-in & 2.24 & 1.37 & 16.31 & 8.00 & $<0.05$ \\
\hline wash-out (x10E-1) & 0.00 & 1,3 & $-3,7$ & 1.1 & $<0.05$ \\
\hline
\end{tabular}

\section{CONCLUSION}

A frame work has been developed to accurately register DCE-MRI data to histology in order to validate MRI derived parametric maps. Non-rigid registration is necessary for exact co-localization of DCE-MRI data with histology. However, artifacts due to cutting (tearing and shattering) and alterations in morphology (shrinkage) hamper correct colocalization. Therefore, the DCE-MRI validation has to be limited to regions where registration was successful.

Preliminary results for the tumor we studied show significant different mean values between vital and non-vital regions for parameter maps calculated with the two-compartment model as well as with semi-quantitative measures. In addition, parameters b, wash-in and AUC90 can discriminate between the different tumor regions. For this tumor, the combination of two well chosen DCE-MRI parameters will enable segmentation into vital and non-vital regions. In the future this study will be expanded using more data and including multiple quantitative DCE-analysis methods, different AIF estimation procedures and calibration methods, and different histology stains. Also a method for segmenting vital and non-vital tumor tissue will be developed.

\section{ACKNOWLEDGEMENT}

This work was funded by the Dutch Cancer Society - KWF (grant no. 2008-4037).

\section{REFERENCES}

[1] Yankeelov, T. E. and Gore, J. C., "Dynamic Contrast Enhanced Magnetic Resonance Imaging in Oncology: Theory, Data Acquisition, Analysis, and Examples, " Curr. Med. Imaging Rev. 3(2), 91-107 (2009).

[2] Egeland, T. A., Gaustad, J. V., Benjaminsen, I. C., Hedalen, K., Mathiesen, B. and Rofstad, E. K., "Assessment of fraction of hypoxic cells in human tumor xenografts with necrotic regions by dynamic contrast-enhanced MRI," Radiat. Res. 169(6), 689-699 (2008).

[3] Egeland, T. A., Simonsen, T. G., Gaustad, J. V., Gulliksrud, K., Ellingsen, C. and Rofstad, E. K., "Dynamic contrast-enhanced magnetic resonance imaging of tumors: preclinical validation of parametric images," Radiat. Res. 172(3), 339-347 (2009).

[4] Gaustad, J. V., Brurberg, K. G., Simonsen, T. G., Mollatt, C. S. and Rofstad, E. K., "Tumor vascularity assessed by magnetic resonance imaging and intravital microscopy imaging," Neoplasia 10(4), 354-362 (2008).

[5] Gulliksrud, K., Brurberg, K. G. and Rofstad, E. K., "Dynamic contrast-enhanced magnetic resonance imaging of tumor interstitial fluid pressure," Radiother. Oncol. 91(1), 107-113 (2009). 
[6] McPhail, L. D., McIntyre, D. J., Ludwig, C., Kestell, P., Griffiths, J. R., Kelland, L. R. and Robinson, S. P., "Rat tumor response to the vascular-disrupting agent 5,6-dimethylxanthenone-4-acetic acid as measured by dynamic contrast-enhanced magnetic resonance imaging, plasma 5-hydroxyindoleacetic acid levels, and tumor necrosis," Neoplasia 8(3), 199-206 (2006).

[7] Atkin, G., Taylor, N. J., Daley, F. M., Stirling, J. J., Richman, P., Glynne-Jones, R., d’Arcy, J. A., Collins, D. J. and Padhani, A. R., "Dynamic contrast-enhanced magnetic resonance imaging is a poor measure of rectal cancer angiogenesis," Br. J. Surg. 93(8), 992-1000 (2006).

[8] Jackson, A. S., Reinsberg, S. A., Sohaib, S. A., Charles-Edwards, E. M., Jhavar, S., Christmas, T. J., Thompson, A. C., Bailey, M. J., Corbishley, C. M., Fisher, C., Leach, O. M. and Dearnaley, D. P., "Dynamic contrastenhanced MRI for prostate cancer localization," Br. J. Radiol. 82(974), 148-156 (2009).

[9] Alic, L., Haeck, J. C., Klein, S., Bol, K., van Tiel, S. T., Wielepolski, P. A., Bijster, M., Niessen, W. J., Bernsen, M., Veenland, J. F. and de Jong, M., "Multi-modal imag registration: matching MRI with histology," Proc. SPIE 7626, (2010).

[10] Klein, S., Staring, M., Murphy, K., Viergever, M. A. and Pluim, J. P., "Elastix: a toolbox for intensity-based medical image registration," IEEE transactions on medical imaging 29(1), 196-205 (2010).

[11]Deoni, S. C., Rutt, B. K. and Peters, T. M., "Rapid combined T1 and T2 mapping using gradient recalled acquisition in the steady state," Magn. Reson. Med. 49(3), 515-526 (2003).

[12] Brix, G., Semmler, W., Port, R., Schad, L. R., Layer, G. and Lorenz, W. J., "Pharmacokinetic parameters in CNS Gd-DTPA enhanced MR imaging," J. Comput. Assist. Tomogr. 15, 621-628 (1991).

[13] Hayton, P., Brady, M., Tarassenko, L. and Moore, N., "Analysis of dynamic MR breast images using a model of contrast enhancement," Medical image analysis 1(3), 207-224 (1997). 